\title{
Seismological demonstration of perpendicular density structuring in the solar corona
}

\author{
T. Van Doorsselaere, C. S. Brady, E. Verwichte, and V. M. Nakariakov
}

\begin{abstract}
Centre for Fusion, Space and Astrophysics, Physics Department, University of Warwick, Coventry CV4 7AL, UK e-mail: [t.van-doorsselaere;c.s.brady;v.nakariakov; erwin.verwichte]@warwick.ac.uk Received 23 July 2008 / Accepted 18 September 2008
\end{abstract}

\section{ABSTRACT}

\begin{abstract}
The peculiarities of the propagating transverse waves observed in the solar corona with the Coronal Multi-channel Polarimeter (CoMP) indicate the existence of fine field structuring in the coronal density. We present results of numerical simulations studying the evolution of a localised transverse magneto-hydrodynamic wave in a uniform magnetic field. We consider two initial low plasma-beta equilibria: one with a homogeneous density, and one with a field-aligned dense structure (such as a loop or a plume). The perpendicular localisation of the wave strongly determines the angular distribution of the energy propagation. If the perpendicular scale of the wave is significantly smaller than the parallel scale (e.g. wavelength), as established by CoMP, the wave develops as an oblique fast magneto-acoustic wave. In an unstructured medium, the energy of such a wave is transferred mainly across the magnetic field. However, it is possible to channel the energy of the transverse wave along the magnetic field in the presence of a field-aligned density enhancement. We conclude that the CoMP results provide an independent seismological proof that the corona is structured in density in the perpendicular direction.
\end{abstract}

Key words. Sun: corona - Sun: oscillations - Sun: magnetic fields

\section{Introduction}

Since the Skylab observations, we know that the corona is highly structured (Vaiana et al. 1973). This structuring was confirmed using space-bourne EUV imaging telescopes, such as SOHO and TRACE (e.g. Aschwanden 2005, and references therein). However, it is difficult to assess the true nature of this structuring (e.g. temperature or density) due to the complex dependency of the EUV emission and instrument bandpass on temperature and density (e.g. Reale \& Peres 2000, and references therein). Recently, it has become possible using spectroscopy to create temperature maps (Reale et al. 2007). However, imaging or spectroscopic data do not easily allow one to determine the magnetic field structuring. MHD waves allow us to indirectly assess the nature of structuring. Propagating slow magneto-acoustic waves provide information about temperature structuring (Robbrecht et al. 2001; King et al. 2003) but not density or magnetic field. Propagating fast magneto-acoustic waves are strongly dependent on density and magnetic field strength and only very weakly dependent on temperature. Due to the low coronal plasma beta, the fast waves are most sensitive to the density structuring.

In 2007, it was discovered by Tomczyk et al. (2007) using CoMP that the corona is filled with low-amplitude $\left(\lesssim 1 \mathrm{~km} \mathrm{~s}^{-1}\right)$ transverse waves propagating along the magnetic field with a projected speed of 1-4 Mm/s. The spatial correlation lengths of the waves were 9 and $45 \mathrm{Mm}$ across and along the magnetic field, respectively. The waves were only observed in velocity, but not in intensity or line-width. The observed waves were interpreted by Van Doorsselaere et al. (2008a) as fast magnetoacoustic kink waves.

Currently, a similar debate is being conducted about the nature of transverse waves observed in spicules (Zaqarashvili \& Skhirtladze 2008, and references therein).
The presence of these low-amplitude waves in the solar atmosphere is an important discovery, as they may play a role in the coronal heating problem. Moreover, the strongest potential of these waves comes from the perspective of coronal seismology.

Coronal seismology is the technique where observed wave properties are compared to existing models to derive physical quantities difficult to measure in the corona (Nakariakov \& Verwichte 2005). The idea of coronal seismology was originally proposed by Uchida (1970) and Roberts et al. (1984). However, the idea could not be used until the launch of high resolution space telescopes, such as TRACE. Since then, the technique has been successfully used to estimate the magnetic field (Nakariakov \& Ofman 2001; Verwichte et al. 2004; Ofman \& Wang 2008; Van Doorsselaere et al. 2008b), the density scale heights (Andries et al. 2005) and Alfvén transit times (Arregui et al. 2007).

In this Letter, we study numerically the time evolution of transverse velocity pulses resembling the observed waves (Sect. 2). We investigate the effect of the presence of wave guides on the propagation of wave energy along the magnetic field. We discuss the implications of our simulations and use coronal seismology to prove that the corona observed by Tomczyk et al. (2007) has perpendicular structuring (Sect. 3). We demonstrate that perturbations with the observed properties are not able to propagate along the field, as the observations show. We demonstrate that the observational findings can be met only if there are magnetic field aligned density structures in the corona.

\section{Numerical experiments}

We have set up MHD simulations using Lare2D (Arber et al. 2001). We use a homogeneous vertical magnetic field (in the $z$-direction) and a low gas pressure (the plasma beta $\beta=0.001$ ) 

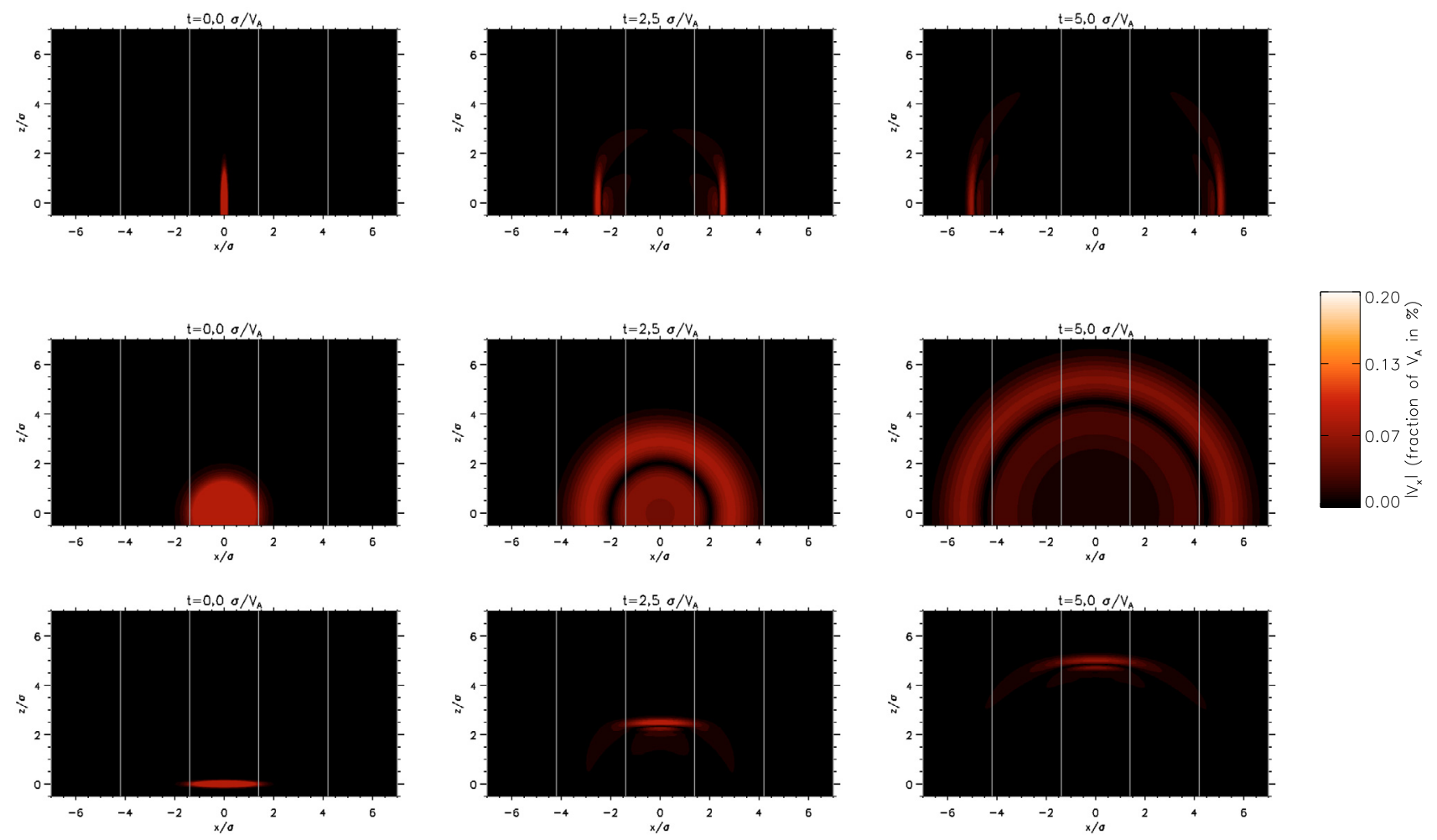

Fig. 1. The evolution of a transverse velocity pulse for three different aspect ratios of the initial pulse. The top row corresponds to an aspect ratio of $C=0.1$, the middle row to $C=1$ and the bottom row to $C=10$. The colour coding displays the absolute value of $V_{x}$ as a fraction of the Alfvén speed in percent. The uniform magnetic field is directed vertically, and is indicated with vertical gray lines.

in the numerical experiments. In the simulations, we set up an initial velocity perturbation $\left(V_{x}\right)$ polarised perpendicular to the magnetic field and the ignorable direction (the $y$-direction). A single pulse is considered to demonstrate clearly how waves are guided through the medium. The pulse is given a finite extent in both the $x$ - and $z$-direction, which is representative of the observed wave packets.

We vary the density between two setups. In the first setup, we impose a homogeneous density. In the second setup, we put a density enhancement along the magnetic field with a Gaussian density profile across the magnetic field.

We have used closed boundary conditions, symmetrically placed around $(x=0, z=0)$. The boundary conditions are not relevant to the simulations. They only play a role at the very end $\left(t V_{A} / \sigma>4.2\right)$ of the simulations with the density structuring (Fig. 2). Their influence can be observed as reflected waves in the right panel of Fig. 2, and a disturbance in the dashed curve of Fig. 3.

\subsection{Homogeneous plasma}

In this subsection, we consider transverse velocity pulses in a homogeneous magnetic field and density. The initial velocity pulse $\boldsymbol{V}=\left(V_{x}, 0,0\right)$ is given a Gaussian shape both along the magnetic field and across:

$V_{x}(x, z)=A V_{A} \exp \left\{-\left(\frac{x}{\sigma_{\perp}}\right)^{2}\right\} \exp \left\{-\left(\frac{z}{\sigma_{\|}}\right)^{2}\right\}$,

where $A=0.001$ is the initial amplitude, $V_{A}$ the Alfvén speed, and $\sigma_{\perp, \|}$ the extent of the pulse in the direction across and along the field, respectively. We have taken the amplitude of the pulse to be small enough to eliminate all non-linear effects.
From linearised MHD wave theory, we know that a wave pulse of infinite extent in the $x$-direction $\left(\sigma_{\perp} \rightarrow \infty\right)$ will be a shear Alfvén wave. This case corresponds to a one dimensional configuration where fast magneto-acoustic and Alfvén waves are degenerate. The pulse will split in two equal pulses travelling along the magnetic field with the Alfvén speed. We prove here that this does not remain true when a finite extent of the pulse in the perpendicular $(x)$ direction is considered.

In Fig. 1 we present the upper half of the computational domain with the evolution of the initial pulse for different extents of the pulse in the $x$-direction. The bottom half of the simulation is not displayed as it is a mirror image of the shown results. We take $\sigma_{\perp}=C \sigma_{\|}$, where $C=0.1$ for the top row, $C=1$ for the middle row and $C=10$ for the bottom row. We have normalised all spatial quantities to $\max \left(\sigma_{\perp}, \sigma_{\|}\right)$and all velocities to $V_{A}(0,0)$. In the bottom row of Fig. 1 , it can be seen that for the case with a high aspect ratio, the pulse evolves as expected from the linearised MHD wave theory. Two plane wave pulses develop and travel in opposite directions along the magnetic field with the Alfvén velocity. The amount of kinetic energy that travels along the magnetic field is much larger than the amount of energy that travels across.

For an aspect ratio of 1 (middle row), the pulse develops into an almost circular wave front, which travels across the magnetic field as well. This means that the transverse pulse takes on a fast magneto-acoustic character. In this case, the energy propagates in all directions equally.

A pulse elongated along the magnetic field (top row) develops mainly into semi-circular wave fronts travelling across the magnetic field, with hardly any energy travelling along the magnetic field.

It can be concluded from these simulations that it is impossible to have energy propagating along the magnetic field when the 

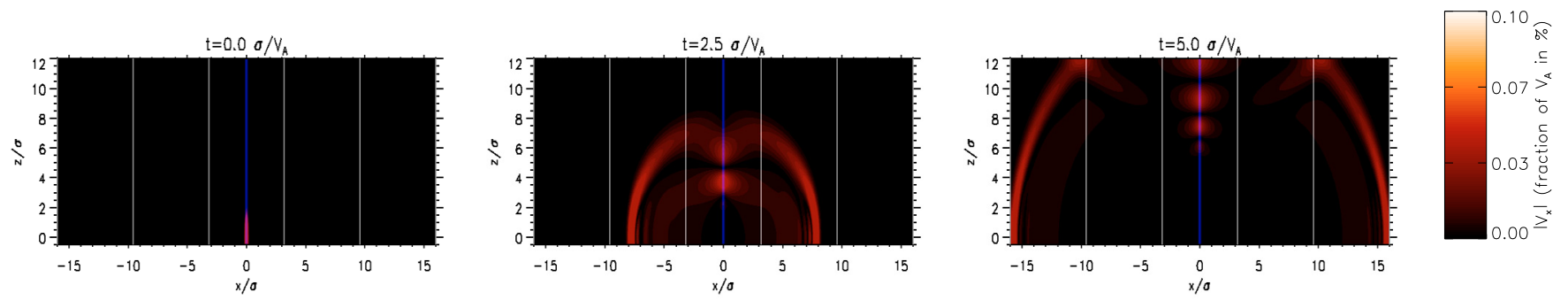

Fig. 2. The evolution of a transverse velocity pulse of aspect ratio $C=0.1$ in a homogeneous magnetic field and a density enhancement aligned with the homogeneous vertical magnetic field. The colour coding shows the absolute value of $V_{x}$ as a fraction of the Alfvén speed in percent. The uniform magnetic field is indicated with vertical gray lines. The density enhancement is shown with a vertical blue line.

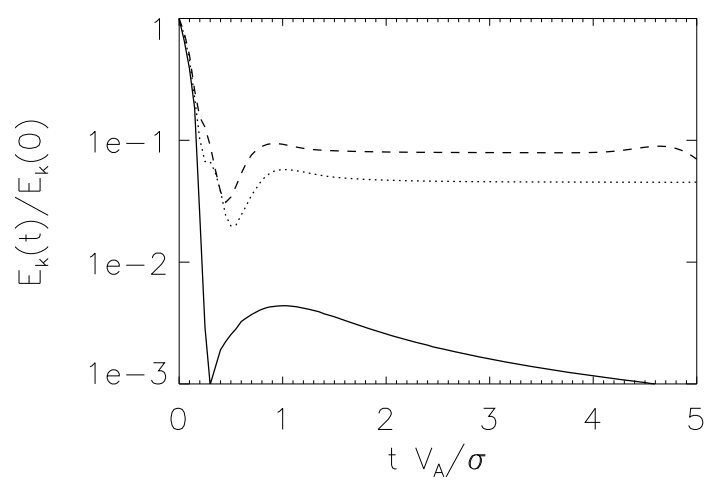

Fig. 3. The fraction of the total kinetic energy $E_{\mathrm{k}}(t)$ still inside the tube boundaries $\left(-\sigma_{\rho} \leq x \leq \sigma_{\rho}\right)$ as a function of time. The full line shows the energy for a homogeneous medium, while the dotted (resp. dashed) line shows the energy for a medium with a density enhancement of $\rho_{0} / \rho_{\mathrm{e}}=6$ (resp. $\left.\rho_{0} / \rho_{\mathrm{e}}=10\right)$.

transverse velocity pulse is sufficiently elongated along the magnetic field. As this is the case in the observed waves (Tomczyk et al. 2007), we can conclude that the observed corona cannot be modelled by a homogeneous plasma.

\subsection{Plasma with wave guide}

Consider a transverse velocity pulse with a shape elongated along the magnetic field $(C=0.1)$ in a medium with a density enhancement aligned with the magnetic field. We take the density $\rho$ as

$\rho(x, z)=\rho_{0} \exp \left\{-\left(\frac{x}{\sigma_{\rho}}\right)^{2}\right\}+\rho_{\mathrm{e}}$,

where $\rho_{0}$ is the density at the centre of the enhancement, which is equal to the density considered in the homogeneous case (Sect. 2.1), and $\sigma_{\rho}=0.15 \sigma_{\|}$describes the horizontal extent of the density enhancement and $\rho_{\mathrm{e}}$ is the external density.

The results of this simulation are shown in Fig. 2. It is clear that, as in the homogeneous case, a large fraction of the initial pulse energy travels outwards in a ring-like wavefront. However, a significant fraction of the kinetic energy travels along the magnetic field with the Alfvén speed. This wave travelling along the magnetic field can be identified as the fast magneto-acoustic kink wave (Edwin \& Roberts 1982).

In the right hand panel of Fig. 2, it can be seen that the initial pulse undergoes damping and wave dispersion along the magnetic field. This was predicted theoretically and numerically in Nakariakov et al. (2004).
To show the confinement of energy more quantitatively, we calculated the fraction of the total kinetic energy still inside the domain $-\sigma_{\rho} \leq x \leq \sigma_{\rho}$, and plotted it against time (Fig. 3) for the homogeneous case (full line) and the structured case (with $\rho_{0} / \rho_{\mathrm{e}}=6,10$ : dotted and dashed line). It can be seen from the figure that most of the energy leaks away from the considered domain during the initial phase with transient behaviour. The initial dip in the confined energy is caused by the displacement of the slab outside its equilibrium position.

For the structured medium at least an order of magnitude more energy stays inside the higher density slab and travels along the magnetic field. Moreover, we can see that the confined energy for the structured cases settle into an equilibrium. In the homogeneous case, the energy continues to leak out of the considered region with a $1 / r$-dependency, as can be expected from the linear wave theory.

Limiting the simulations to $2 \mathrm{D}$ does not have a great impact on the qualitative results. In $3 \mathrm{D}$, it can be expected that a lower energy confinement is reached in the $3 \mathrm{D}$ structure, as there is more "room" available to have energy leak away (spherical vs. cylindrical waves). Another change would be that the energy would leak away more rapidly $\left(1 / r^{2}\right)$ in the homogeneous case (Ruderman \& Roberts 2002; Mikhalyaev \& Solov'ev 2005; Terradas et al. 2006). In the discussed context, it means that the wave energy carried upwards along the field decreases even more rapidly.

\section{Application and coronal seismology}

The main conclusion from the above results is that a transverse velocity pulse elongated along the magnetic field can only propagate along the magnetic field if a wave guide aligned with the magnetic field is present. We can apply this knowledge to the observational results of Tomczyk et al. (2007). They used CoMP to observe the corona in white light emission. They found lineof-sight velocity waves propagating along the magnetic field. The perpendicular extent in the plane of the sky of the observed waves was very small when compared to their extent along the magnetic field (see their Fig. 3). From their figure, the extent along the magnetic field was estimated to be $45 \mathrm{Mm}$, whereas the perpendicular extent was measured to be $9 \mathrm{Mm}$. Since there are no extended photospheric drivers, it is reasonable to say that the line-of-sight extent of the pulse is of the same order of magnitude as the perpendicular extent, i.e. $9 \mathrm{Mm}$. Tomczyk et al. (2007) found that the velocity pulses travelled along the magnetic field and did not exhibit significant damping.

We can relate our simulation results to these observational results directly. From our simulations (especially Fig. 3), we concluded that propagation of wave energy along the magnetic 
field is only possible if a wave guide aligned with the magnetic field is present. We can thus establish that the observed fast magneto-acoustic kink waves can only exist if perpendicular structuring is present in the CoMP observations, even though it is not directly observed. The most likely reason for these structures not being observed directly in the white light emission is the line-of-sight integration (November \& Koutchmy 1996).

This coronal seismological proof corroborates the presence of a multitude of coronal density structuring visible in images taken with imaging telescopes. It was impossible to conclusively prove this perpendicular structuring from the imaging results alone, because of the complicated dependence of the coronal emission upon temperature and density, and line-of-sight effects.

Our simulations prove that the important parameter is a minimum in the Alfvén speed. This can be caused by a minimum in the magnetic field, or a density enhancement. Because the corona is a low beta plasma, we can expect that the magnetic field fills the corona almost homogeneously, without being hampered by the gas forces. We must conclude that the structuring is due to density structuring.

The perpendicular extent of the density structures or the extent of the initial driver can be estimated from the width of the observed wave. In our simulations, we have varied the width of the initial pulse, while keeping the width of the wave guide constant. If $\sigma_{\perp}<\sigma_{\rho}$, the pulse will quickly fill the whole slab volume and then propagate as a guided wave. On the other hand, if $\sigma_{\perp}>\sigma_{\rho}$, the excess energy not initially in the slab will rapidly leak away and only the energy in the slab will be guided along the magnetic field.

In Fig. 2 it can be seen that the velocity profile extends significantly further than the loop width. Also, if multiple density structures are included, the width of the energy travelling along the field is determined by the width of the initial pulse, if it extends over multiple strands. These two facts lead us to the conclusion that the width of the observed wave is an upper boundary for the maximum of the width of the driver and the width of the wave guide. This agrees with simple geometric estimates. Take a photospheric magnetic field of $1000 \mathrm{G}$ and coronal fields of $10 \mathrm{G}$. That means that the photospheric magnetic field element with a typical horizontal size of $1 \mathrm{Mm}$ has expanded linearly by a factor of 10 . The expected density structures would have a width of $10 \mathrm{Mm}$, which agrees very well with the measured wave extent of Tomczyk et al. (2007).

We would like to point out that the discussed results do not contradict the observational findings of shear Alfvén waves, e.g. in the solar wind (Tu \& Marsch 1995). If the perpendicular extent of the transverse (Alfvénic) perturbation is much longer than the parallel extent, the wave propagates predominantly as a shear Alfvén wave (see bottom row in Fig. 1).

The 2D model employed here contains all essential physics, except for the Alfvén continuum and the associated physical phenomena, such as resonant absorption and phase mixing (Goossens et al. 1995; Goedbloed \& Poedts 2004; Tsiklauri \& Nakariakov 2002), and for the stronger leakage discussed above. The collective kink mode is not an artifact of the cylindrical symmetry, and has been reproduced in complex plasma structures (e.g. Ruderman 2003; Terradas et al. 2008).

In $3 \mathrm{D}$, a pulse localised in three directions will naturally excite an Alfvénic component. However, the amount of energy in this component will be negligible for a pulse elongated along the magnetic field. We can conclude that the above picture will remain true in a $3 \mathrm{D}$ configuration.

\section{Conclusions}

We have studied the time evolution of a velocity pulse transverse to the magnetic field which is localised both perpendicular to and along the magnetic field. We have considered a medium with a homogeneous plasma and with a density enhancement along the magnetic field. We have concluded that it is only possible to guide a significant amount of the initial pulse energy along the magnetic field in the presence of the density enhancement.

We have compared the numerical results to the observational results of Tomczyk et al. (2007). These waves are localised across and along the magnetic field and are seen to propagate along the magnetic field. According to our numerical experiments, this can only be the case if perpendicular structuring is present.

This seismological proof of perpendicular structuring in the corona underlines that the structuring observed with imaging telescopes is not an artifact of the complicated dependence of the coronal emission upon the temperature and density. It is rather the result of density structuring across the magnetic field.

The observed waves are omni-present and are guided along the field everywhere. Our result confirms that perpendicular structuring of the density is present everywhere in the corona.

The perpendicular extent of the observed wave can be used as an estimate for the maximum of the size of the driver and of the perpendicular size of the supporting wave guide.

Acknowledgements. This research was supported by a Marie Curie Intra European Fellowship within the 7th European Community Framework Programme, and STFC. It was inspired by the workshop organised by ISSI.

\section{References}

Andries, J., Arregui, I., \& Goossens, M. 2005, ApJ, 624, L57

Arber, T. D., Longbottom, A. W., Gerrard, C. L., \& Milne, A. M. 2001, J. Comput. Phys., 171, 151

Arregui, I., Andries, J., Van Doorsselaere, T., Goossens, M., \& Poedts, S. 2007, A\&A, 463, 333

Aschwanden, M. J. 2005, Physics of the Solar Corona, An Introduction with Problems and Solutions, 2nd edn. (Pour la Science)

Edwin, P. M., \& Roberts, B. 1982, Sol. Phys., 76, 239

Goedbloed, J. P., \& Poedts, S. 2004, Principles of magnetohydrodynamics (Cambridge University Press)

Goossens, M., Ruderman, M. S., \& Hollweg, J. V. 1995, Sol. Phys., 157, 75

King, D. B., Nakariakov, V. M., Deluca, E. E., Golub, L., \& McClements, K. G. 2003, A\&A, 404, L1

Mikhalyaev, B. B., \& Solov'ev, A. A. 2005, Sol. Phys., 227, 249

Nakariakov, V. M., \& Ofman, L. 2001, A\&A, 372, L53

Nakariakov, V. M., \& Verwichte, E. 2005, Living Rev. Solar Phys., 2, 3

Nakariakov, V. M., Arber, T. D., Ault, C. E., et al. 2004, MNRAS, 349, 705

November, L. J., \& Koutchmy, S. 1996, ApJ, 466, 512

Ofman, L., \& Wang, T. J. 2008, A\&A, 482, L9

Reale, F., \& Peres, G. 2000, ApJ, 528, L45

Reale, F., Parenti, S., Reeves, K. K., et al. 2007, Science, 318, 1582

Robbrecht, E., Verwichte, E., Berghmans, D., et al. 2001, A\&A, 370, 591

Roberts, B., Edwin, P. M., \& Benz, A. O. 1984, ApJ, 279, 857

Ruderman, M. S. 2003, A\&A, 409, 287

Ruderman, M. S., \& Roberts, B. 2002, ApJ, 577, 475

Terradas, J., Oliver, R., \& Ballester, J. L. 2006, ApJ, 642, 533

Terradas, J., Arregui, I., Oliver, R., et al. 2008, ApJ, 679, 1611

Tomczyk, S., McIntosh, S. W., Keil, S. L., et al. 2007, Science, 317, 1192

Tsiklauri, D., \& Nakariakov, V. M. 2002, A\&A, 393, 321

Tu, C.-Y., \& Marsch, E. 1995, Space Sci. Rev., 73, 1

Uchida, Y. 1970, PASJ, 22, 341

Vaiana, G. S., Krieger, A. S., \& Timothy, A. F. 1973, Sol. Phys., 32, 81

Van Doorsselaere, T., Nakariakov, V. M., \& Verwichte, E. 2008a, ApJ, 676, L73

Van Doorsselaere, T., Nakariakov, V. M., Young, P. R., \& Verwichte, E. 2008b, A\&A, 487, L17

Verwichte, E., Nakariakov, V. M., Ofman, L., \& Deluca, E. E. 2004, Sol. Phys., 223, 77

Zaqarashvili, T. V., \& Skhirtladze, N. 2008, ApJ, 683, L91 